\title{
Efficacy and safety of cyclophosphamide in anthracycline- and taxane-based neoadjuvant chemotherapy in breast cancer: a meta-analysis
}

\author{
Yi-Kun Kang ${ }^{1 \wedge}$, Yi-Ran Si ${ }^{1}$, Guang-Yu An ${ }^{2}$, Peng Yuan ${ }^{1}$ \\ ${ }^{1}$ Department of VIP Medical Services, National Cancer Center/ National Clinical Research Center for Cancer/ Cancer Hospital, Chinese Academy \\ of Medical Sciences and Peking Union Medical College, Beijing, China; ${ }^{2}$ Department of Oncology, Beijing Chao-Yang Hospital, Capital Medical \\ University, Beijing, China \\ Contributions: (I) Conception and design: YK Kang, P Yuan, GY An; (II) Administrative support: P Yuan, GY An; (III) Provision of study materials \\ or patients: YK Kang, P Yuan; (IV) Collection and assembly of data: YK Kang, P Yuan; (V) Data analysis and interpretation: YK Kang, P Yuan; (VI) \\ Manuscript writing: All authors; (VII) Final approval of manuscript: All authors. \\ Correspondence to: Peng Yuan, MD. Department of VIP Medical Services, National Cancer Center/ National Clinical Research Center for Cancer/ \\ Cancer Hospital, Chinese Academy of Medical Sciences and Peking Union Medical College, Beijing 100021, China. Email: yuanpeng01@hotmail.com; \\ Guang-Yu An, MD. Department of Oncology, Beijing Chao-Yang Hospital, Capital Medical University, Beijing 100020, China. Email: agybjcy@163.com.
}

Background: Our study aimed to compare the efficacy and safety of anthracycline plus taxane (AT)-based neoadjuvant chemotherapy (NAC) with or without cyclophosphamide in the treatment of breast cancer.

Methods: We searched PubMed, Embase, Web of Science and the Cochrane Library for randomized controlled studies comparing the efficacy and safety of AT-based NAC with or without cyclophosphamide in breast cancer patients.

Results: Four eligible studies with 2,302 individuals were ultimately included in the quantitative analysis. After applying the AT-based NAC regimen, the overall rates of pathologic complete response (pCR) and breast conserving surgery in all included subjects were $26.5 \%$ and $70.6 \%$, respectively. The rates of pCR [risk ratio (RR): 1.35; 95\% CI: 0.75, 2.45; P=0.32], breast-conserving surgery (RR: 1.07; 95\% CI: 0.97, 1.19; $\mathrm{P}=0.17$ ) and clinical response (RR: 1.08; 95\% CI: 0.97, 1.19; $\mathrm{P}=0.15)$ in patients in the cyclophosphamide group were similar to those in the control group. However, participants in the cyclophosphamide group had a lower no clinical response rate than those in the control group (RR: 0.72; 95\% CI: 0.60, 0.87; P<0.001). Subjects in the cyclophosphamide group had significantly lower rates of infection (RR: 0.57; 95\% CI: 0.41, $0.79 ; \mathrm{P}<0.001)$ and diarrhea (RR: 0.46; 95\% CI: 0.30, 0.68; $\mathrm{P}<0.001)$ and higher rates of thrombocytopenia (RR: 3.38; 95\% CI: 1.96, 5.84; $\mathrm{P}<0.001$ ), sensory/motor neuropathy (RR: 1.57; 95\% CI: 1.03, 2.39; P=0.03) and nausea/vomiting (RR: $1.51 ; 95 \% \mathrm{CI}: 1.11,2.06 ; \mathrm{P}=0.009$ ) than those in the control group.

Conclusions: The AT-based NAC regimen with or without cyclophosphamide had similar clinical outcomes in breast cancer patients. The addition of cyclophosphamide might increase the risks of thrombocytopenia, sensory/motor neuropathy and nausea/vomiting.

Keywords! Neoadjuvant chemotherapy (NAC); cyclophosphamide; anthracycline; taxane; breast cancer

Submitted Jul 03, 2020. Accepted for publication Nov 27, 2020.

doi: $10.21037 / \mathrm{gs}-20-593$

View this article at: http://dx.doi.org/10.21037/gs-20-593

^ ORCID: 0000-0002-9898-3783. 


\section{Introduction}

Breast cancer is the most frequently diagnosed cancer as well as the leading cause of cancer-related death among females throughout the world, with 1.67 million new cases and 0.52 million deaths in 2012 (1-3). In 2018, the estimated number of new cases increased to 2.08 million globally, with 0.62 million deaths $(1,2)$. Although the mortality rate of breast cancer has decreased with improvements in diagnosis and intervention, breast cancer remains the leading cause of cancer-related death among women in developed counties (3).

Neoadjuvant chemotherapy (NAC), which was initially used only for locally advanced cancers, has now become more common for patients with operable disease (4). It has been reported that NAC is effective in downstaging a tumor, reducing tumor size, improving the chance of undergoing surgery, evaluating the sensitivity of a tumor, and allowing time to fully consider surgical options (5). Recent studies have also indicated NAC as an efficient approach to improve pathological complete response (pCR) and the possibility of breast-conserving surgery in breast cancer patients (6). Therefore, NAC has become an optional first-line surgical therapy for the treatment of breast cancer.

Anthracycline plus taxane (AT)-based chemotherapy is the most common NAC regimen for all early breast cancer subtypes and is associated with high rates of clinical response $(7,8)$. It has been discussed whether cyclophosphamide should be added to AT-based NAC to improve clinical outcomes for breast cancer patients, but the conclusions have not been consistent. Some studies report that cyclophosphamide improves the pCR rate in breast cancer, while others report no differences (9). Although cyclophosphamide has been found to increase toxicity, some studies have also declared that the addition of cyclophosphamide is able to reduce adverse effects associated with NAC (10). Therefore, it remains unclear whether cyclophosphamide should be added to AT-based NAC. Here, we performed a meta-analysis to compare the efficacy and safety of AT-based NAC with or without cyclophosphamide in the treatment of breast cancer. We present the following article in accordance with the PRISMA reporting checklist (available at http://dx.doi. org/10.21037/gs-20-593).

\section{Methods}

\section{Search strategy}

The protocol of this meta-analysis was registered in
PROSPERO website (University of York, York, UK) with a registration number of CRD42020182821. Two authors (YKK and PY) independently searched four bibliography databases including PubMed, Embase, Web of Science and Cochrane Library using following keywords with various combinations: "neoadjuvant chemotherapy", "mammary cancer", "breast cancer", "breast tumor", “cyclophosphamide”, “cytoxan”, "endoxan”, "anthracycline", "adriacin"; "adriamycin", "amycin", "texane", “doxorubicin”, "paclitaxel”, “docetaxel”, "taxinol”. The searches were limited to human studies and the last search date was Mar 31, 2020.

\section{Study selection}

Inclusion criteria were: (I) randomized controlled study comparing the efficacy or safety of AT-based NAC with or without cyclophosphamide in breast cancer patients; (II) the sample size was at least 30 participants in each group; (III) the study presented a clear process of the clinical trial; (IV) accessible to obtain the data; and (V) English-language literature only. And exclusion criteria were: (I) duplicated reports based on the same participants and failed to provide additional information; (II) ongoing studies or unpublished data; (III) the original data were unavailable; and (IV) studies with obvious defects in design or data statistics. The articles were independently screened and selected by two researchers (YKK and PY), and any disagreements between them were resolved through discussion.

\section{Quality assessment and data extraction}

For the articles that passed the primary screening, they were then reviewed by two authors (YKK and PY). They independently assessed the quality of the studies according to the CONSORT (consolidated standard of reporting trial) statement (11). The Cochrane risk of bias was assessed from six aspects: selection bias, performance bias, detection bias, attrition bias, reporting bias, and other bias. Each aspect of bias was classified into three categories (i.e., low, high, and unclear risk bias). The studies with low quality or evident defects in study design were excluded from this meta-analysis. Any disagreements between the two reviewers were resolved by discussion or judged by senior researchers. Then two authors independently extracted the data: (I) basic characteristics of the included studies and participants; (II) the setting of the studies and the management of NAC; (III) main outcomes including the rates of pathologic complete 


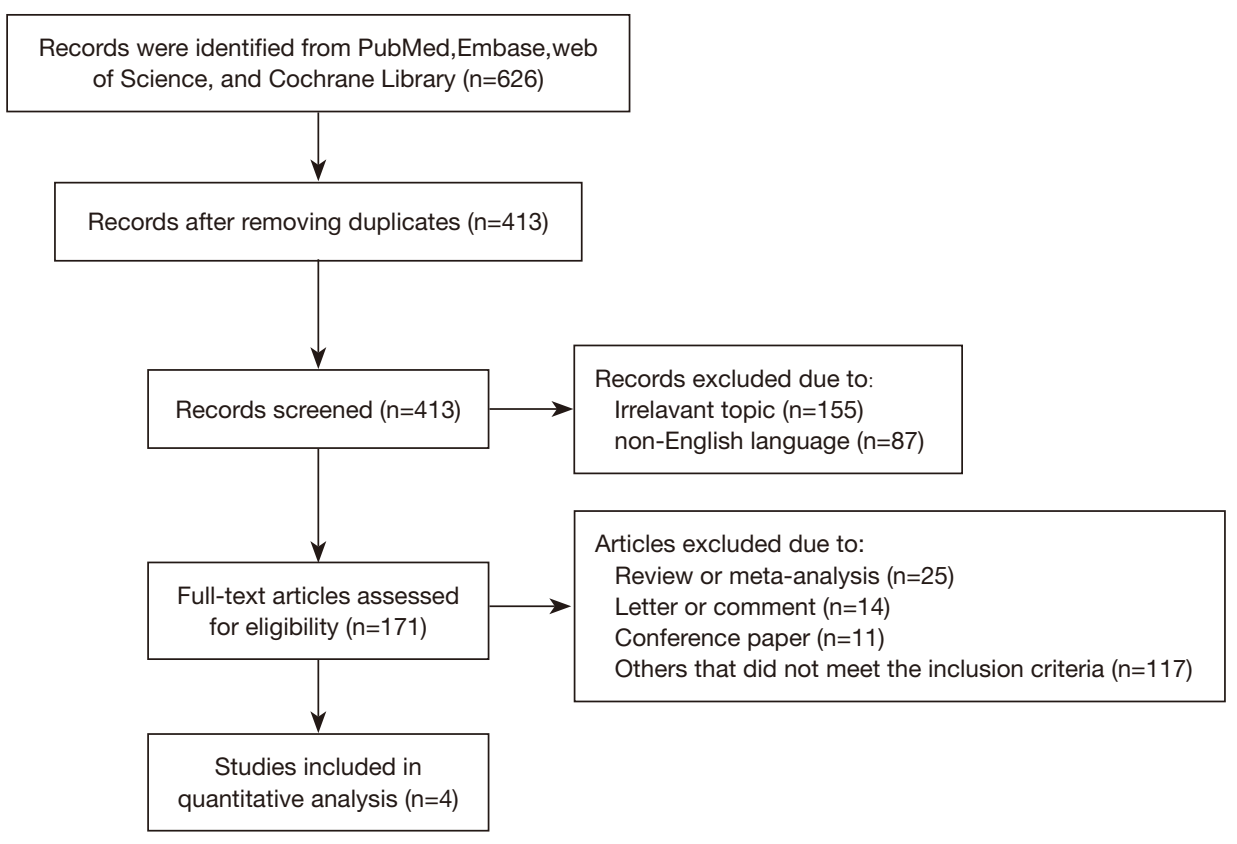

Figure 1 Flow diagram of literature selection (n: number of literature).

response (pCR), breast conserving surgery, clinical response (complete response or partial response) and no clinical response (stable disease or progressive disease); and (IV) second outcomes were adverse effects, including anemia, leucopenia, neutropenia, thrombocytopenia, neutropenic fever, alopecia, infection, fatigue, sensory or motor neuropathy, mucositis, skin changes, loss of appetite, nausea or vomiting, diarrhea, stomatitis, abnormal level of bilirubin, abnormal level of aspartate aminotransferase (AST) and alanine aminotransferase (ALT).

\section{Statistical analysis}

The pooled quantitative analysis was performed by using RevMan 5.3 software (Cochrane Collaboration, Denmark). Risk ratio (RR) with $95 \%$ confidence interval (CI) was calculated for dichotomous variables. Forest plots were used for presenting the pooled clinical outcomes. The statistical heterogeneity among studies was analyzed using the chi-squared test and presented as the I-squared (less than $50 \%$ : low heterogeneity, 50-75\%: moderate heterogeneity, and $\geq 75 \%$ : high heterogeneity). Fixed-effects models were used when the heterogeneity was lower than $50 \%$, otherwise, random-effects models were applied. The publication bias for included studies was evaluated using
Funnel plots. P value lower than 0.05 was considered as statistical significance.

\section{Results}

Figure 1 showed the process of the literature selection. At initial searches, a total of 626 published articles were selected from the four databases mentioned above. After primary screening and removing duplicated paper, 413 articles remained and underwent full-text reviews. After removing the studies with irrelevant topic or in non-English language, 171 articles were potentially eligible. After another round of screening, four eligible studies with 2,302 early-stage breast cancer patients were finally included in the quantitative synthesis (12-15). The assessment of Cochrane risk of bias was illustrated in Figure 2. Most included studies were of high quality with low risk of bias, and high risk of bias was not detected in these studies.

The basic characteristics of the included studies were listed in Table 1. Among the four included studies, three studies were from Germany and the other one was from Korea. Jackisch (12) and von Minckwitz (13) conducted the research based on the same participants in different phases. The former one mainly detected the adverse effects while the latter one revealed the clinical efficacy, and the overlapped 


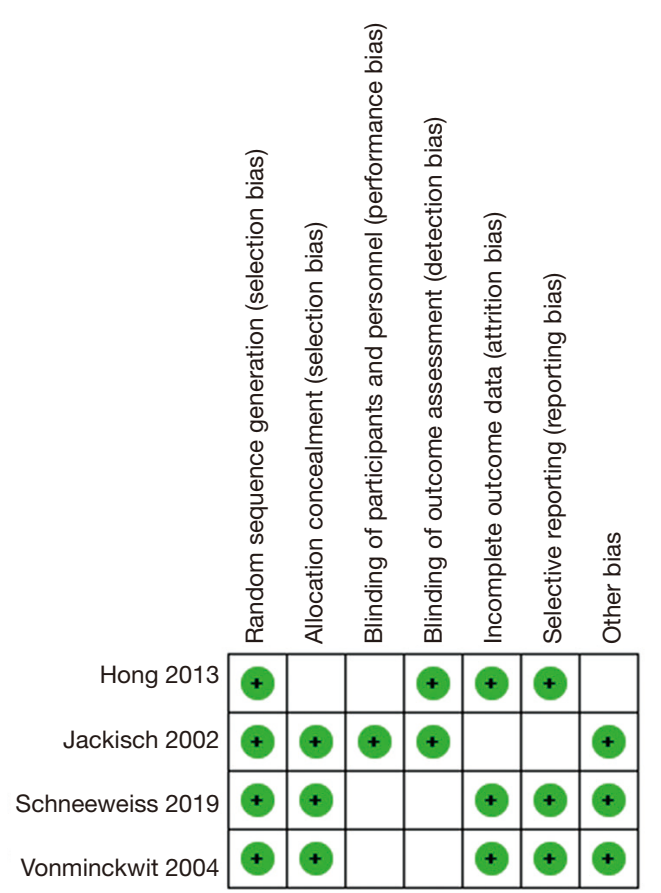

Figure 2 Cochrane bias assessment of the included studies. Green circle: low risk; empty grid: unclear risk.

outcomes between them were not repeatedly included in the analysis. If taking cyclophosphamide group and control group together, the whole pCR rate and breast conserving surgery rate in all included subjects were $26.5 \%$ and $70.6 \%$, respectively, after applying the NAC. Three included studies reported pCR rate according to different molecular types. The pCR rate in hormone receptor (HR) positive subgroup, HR negative subgroup, HER-2 positive subgroup, HER-2 negative subgroup, TNBC subgroup and non-TNBC subgroup was $6.5 \%, 21.8 \%, 57.9 \%, 35.3 \%, 48.9 \%$ and $41.0 \%$, respectively.

In the pooled analysis, patients in the cyclophosphamide group had similar rates of pCR (RR: 1.35 ; 95\% CI: $0.75,2.45$; $\mathrm{P}=0.32 ; \mathrm{I}^{2}=82 \%, \mathrm{P}$ value for heterogeneity $\left.=0.004\right)$, breast conserving surgery (RR: 1.07 ; 95\% CI: 0.97, 1.19; $\mathrm{P}=0.17$; $\mathrm{I}^{2}=55 \%, \mathrm{P}$ value for heterogeneity $\left.=0.11\right)$ and clinical response (RR: 1.08 ; 95\% CI: $0.97,1.19 ; \mathrm{P}=0.15 ; \mathrm{I}^{2}=72 \%$, $\mathrm{P}$ value for heterogeneity $=0.03$ ) compared to those in the control group (Figure 3). However, participants in the cyclophosphamide group had a lower rate of no clinical response rate than those in the control group (RR: 0.72; 95\% CI: 0.60, 0.87; $\mathrm{P}<0.001$; $\mathrm{I}^{2}=0 \%, \mathrm{P}$ value for heterogeneity $\left.=0.71\right)$. In the included studies, only Schneeweiss et al. (15) performed subgroup analysis according to molecular types, and the results showed the cyclophosphamide group had similar pCR rate to the control group no matter in HR positive subgroup or HR negative subgroup or TNBC subgroup $(\mathrm{P}>0.05)$.

The pooled RR of adverse effects in the two groups were summarized in Table 2. Compared to the control group, subjects in the cyclophosphamide group had significantly lower rates of infection (RR: 0.57; 95\% CI: 0.41, 0.79; $\mathrm{P}<0.001 ; \mathrm{I}^{2}=0 \%, \mathrm{P}$ value for heterogeneity $\left.=0.90\right)$ and diarrhea (RR: 0.46; 95\% CI: 0.30, 0.68; $\mathrm{P}<0.001 ; \mathrm{I}^{2}=0 \%$, $\mathrm{P}$ value for heterogeneity $=0.75)$, and higher rates of thrombocytopenia (RR: 3.38 ; 95\% CI: 1.96, 5.84; $\mathrm{P}<0.001$; $\mathrm{I}^{2}=0 \%, \mathrm{P}$ value for heterogeneity $\left.=0.48\right)$, sensory $/$ motor neuropathy (RR: $1.57 ; 95 \%$ CI: $1.03,2.39 ; \mathrm{P}=0.03 ; \mathrm{I}^{2}=0 \%$, $\mathrm{P}$ value for heterogeneity $=0.43$ ) and nausea $/$ vomiting (RR: $1.51 ; 95 \%$ CI: $1.11,2.06 ; \mathrm{P}=0.009 ; \mathrm{I}^{2}=46 \%$, P value for heterogeneity $=0.16$ ). The two groups had similar rates in most adverse events, including anemia, leucopenia, neutropenia, neutropenic fever, alopecia, fatigue, mucositis, skin changes, loss of appetite, stomatitis, abnormal level of bilirubin, abnormal level of AST and ALT (All P value $>0.05$ ).

At last, funnel plots for the RR of pCR rate were calculated to assess the publication bias (Figure S1). No obvious publication bias was found among the included studies in this meta-analysis.

\section{Discussion}

In the current study, the overall rates of pCR and breast-conserving surgery with the AT-based NAC regimen were $26.5 \%$ and $70.6 \%$, respectively, which were consistent with those of former studies. Crown et al. (16) reviewed randomized trials of NAC in breast cancer and reported that the pCR rate of the AT-based regimen was $11.5-26.1 \%$. In the phase III GeparQuattro study, the rates of pCR and breast-conserving surgery with TAC (docetaxel, epirubicin and cyclophosphamide)-based NAC were $22.3 \%$ and $70.1 \%$, respectively. Andrade et al. (17) compared the efficacy between CEF (cyclophosphamide, epirubicin and 5-fluorouracil) and AT (docetaxel and epirubicin) regimens in 316 patients with stage II-III breast cancer. They demonstrated that NAC combined with TA was more effective than NAC combined with CEF in terms of the pCR and clinical response rates as well as the breast-conserving surgery rate. It has been stated that there is synergistic action between anthracycline and taxane by enhancing immune functions. In vitro studies have proven that doxorubicin enhances the function of innate immune cells and cytotoxic T-lymphocytes, which induce the 


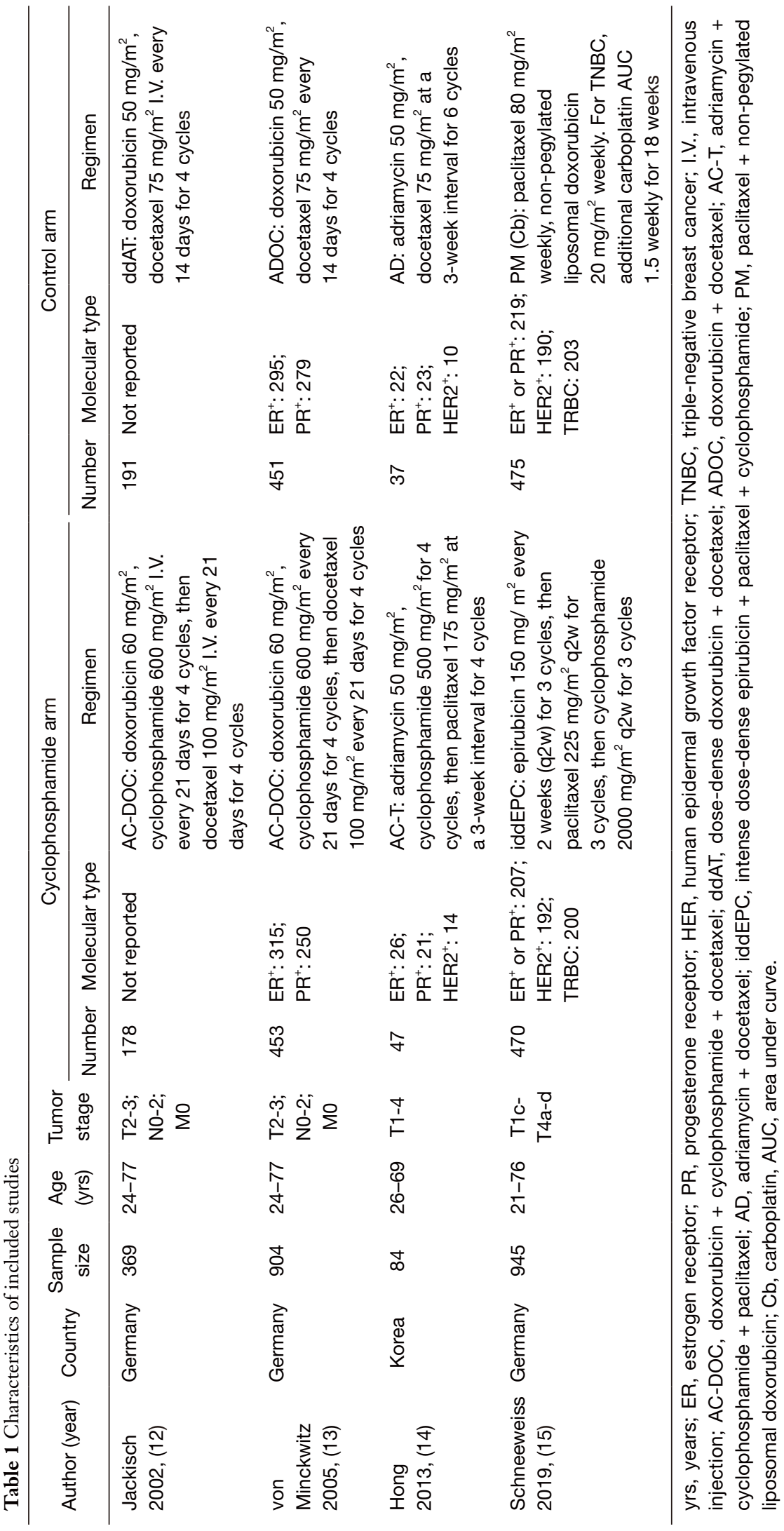




\begin{tabular}{|c|c|c|c|c|c|c|c|c|}
\hline$A$ & Cyclophosphamide & Contro & & & Risk Ratio & & Risk F & Ratio \\
\hline Study or Subgroup & Events & Events & Total & Weight 1 & M-H, Random, $95 \% \mathrm{Cl}$ & & M-H. Rando & lom. $95 \% \mathrm{Cl}$ \\
\hline Hong 2013 & 47 & 4 & 37 & $16.2 \%$ & $1.18[0.36,3.88]$ & & & \\
\hline Schneeweiss 2019 & 470 & 228 & 475 & $45.6 \%$ & $1.01[0.88,1.15]$ & & & \\
\hline Vonminckwitz 2004 & 441 & 31 & 444 & $38.2 \%$ & $2.05[1.36,3.08]$ & & & \\
\hline Total $(95 \% \mathrm{Cl})$ & 958 & & 956 & $100.0 \%$ & $1.35[0.75,2.45]$ & & & \\
\hline Total events & 296 & 263 & & & & & & \\
\hline $\begin{array}{l}\text { Heterogeneity: } \mathrm{Tau}^{2}= \\
\text { Test for overall effect: }\end{array}$ & $\begin{array}{l}0.20 ; C h i^{2}=11.08, d f=2 \\
Z=1.00(P=0.32)\end{array}$ & $(P=0.00$ & 04); $F^{2}=$ & $82 \%$ & & 0.2 & ${ }^{0.5}{ }^{1}$ & $\begin{array}{lc}1 & 1 \\
\text { Favours [Cyclophosphamide] }\end{array}$ \\
\hline B & Cyclophosphamide & Contro & & & Risk Ratio & & Risk $\mathrm{F}$ & Ratio \\
\hline Studv or Subgroup & Events & Events I & Total & Weight & M-H. Random. $95 \% \mathrm{Cl}$ & & M-H. Rando & lom. $95 \% \mathrm{Cl}$ \\
\hline Hong 2013 & 47 & 25 & 37 & $11.0 \%$ & $1.10[0.83,1.46]$ & & & \\
\hline Schneeweiss 2019 & 467 & 323 & 471 & $44.4 \%$ & $1.00[0.92,1.09]$ & & & \\
\hline Vonminckwitz 2004 & 441 & 292 & 444 & $44.6 \%$ & $1.14[1.05,1.24]$ & & & \\
\hline Total $(95 \% \mathrm{Cl})$ & 955 & & 952 & $100.0 \%$ & $1.07[0.97,1.19]$ & & & \\
\hline Total events & 687 & 640 & & & & & & \\
\hline $\begin{array}{l}\text { Heterogeneity: Tau }{ }^{2}= \\
\text { Test for overall effect: }\end{array}$ & $\begin{array}{l}0.00 ; C^{2} i^{2}=4.40, d f=2 \\
Z=1.36(P=0.17)\end{array}$ & $(P=0.11)$ & $; I^{2}=55$ & & & 0.5 & $\begin{array}{l}0.7 \\
\text { Favours [Control] }\end{array}$ & \begin{tabular}{|cc}
1 & 1.5 \\
Favours [Cyclophosphamide]
\end{tabular} \\
\hline C & Cyclophosphamide & Contro & & & Risk Ratio & & Risk $\mathrm{F}$ & Ratio \\
\hline Study or Subgroup & Events & Events 1 & Total & Weight I & M-H, Random, $95 \% \mathrm{Cl}$ & & M-H. Rando & lom, $95 \% \mathrm{Cl}$ \\
\hline Hong 2013 & 47 & 28 & 37 & $13.8 \%$ & $1.07[0.85,1.34]$ & & & \\
\hline Schneeweiss 2019 & 465 & 419 & 474 & $47.4 \%$ & $1.02[0.98,1.07]$ & & & \\
\hline Vonminckwitz 2004 & 430 & 297 & 433 & $38.9 \%$ & $1.15[1.06,1.24]$ & & & \\
\hline Total $(95 \% \mathrm{Cl})$ & 942 & & 944 & $100.0 \%$ & $1.08[0.97,1.19]$ & & & \\
\hline Total events & 797 & 744 & & & & & & \\
\hline $\begin{array}{l}\text { Heterogeneity: Tau }= \\
\text { Test for overall effect: }\end{array}$ & $\begin{array}{l}0.00 ; \mathrm{Chi}^{\mathrm{2}}=7.05, \mathrm{df}=2 \\
Z=1.45(\mathrm{P}=0.15)\end{array}$ & $(P=0.03)$ & $; 1^{2}=72$ & & & 0.7 & ${ }^{0.85}{ }^{1}$ & ${ }^{1} \begin{array}{ccc}1.2 & 1.5 \\
\text { Favours [Cyclophosphamide] }\end{array}$ \\
\hline D & Cyclophosphamide & Contr & & & Risk Ratio & & Risk R & Ratio \\
\hline Stucty or Subgroup & Events & Events & Total & Weight & M-H, Fixed, $95 \% \mathrm{Cl}$ & & M-H, Fixed & d. $95 \% \mathrm{Cl}$ \\
\hline Hong 2013 & 47 & 9 & 37 & $5.0 \%$ & $0.79[0.35,1.78]$ & & & \\
\hline Schneeweiss 2019 & 465 & 55 & 474 & $27.2 \%$ & $0.82[0.56,1.19]$ & & & \\
\hline Vonminckwitz 2004 & 430 & 136 & 433 & $67.7 \%$ & $0.68[0.54,0.86]$ & & & \\
\hline Total $(95 \% \mathrm{Cl})$ & 942 & & 944 & $100.0 \%$ & $0.72[0.60,0.87]$ & & & \\
\hline Total events & 145 & 200 & & & & & & \\
\hline $\begin{array}{l}\text { Heterogeneity: } \mathrm{Chi}^{2}= \\
\text { Test for overall effect }\end{array}$ & $\begin{array}{l}0.70, d f=2(P=0.71) ; 1^{2} \\
Z=3.34(P=0.0008)\end{array}$ & $=0 \%$ & & & & 0.2 & $\begin{array}{c}0.5 \\
\text { Favours [Control] }\end{array}$ & Favours [Cycloph \\
\hline
\end{tabular}

Figure 3 Forest plot showing the risk ratio (RR) with 95\% confidence interval (CI) of pCR rate (A), breast conserving rate (B), clinical response rate $(\mathrm{C})$, and no clinical response rate (D). pCR, pathologic complete response.

immunogenic death of cancer cells (18). Paclitaxel is also able to enhance the antitumor activity of lymphocytes by inducing various cytokines (19). Therefore, chemotherapy combined with doxorubicin and paclitaxel can activate and increase the number of $\mathrm{T}$ lymphocytes in the peripheral blood of breast cancer patients (20).

Among the included studies, only von Minckwitz et al. (13) analyzed the pCR rate of AT-based NAC stratified by molecular subtypes. The hormone receptor status was determined in 783 subjects, and the pCR rates in $\mathrm{HR}^{+}$and HR $^{-}$breast cancers were $6.2 \%$ and $22.8 \%$, respectively. The difference in clinical outcomes between subtypes has been investigated and may be attributed to genetic factors. In triple-negative breast cancer (TNBC), an AT-based regimen is standard. Bignon et al. (21) showed a high pCR rate after NAC in BRCA-mutated TNBC, with a rate of $38.3 \%$ among BRCA1 mutation carriers and $66 \%$ among BRCA2 mutation carriers. Another study explored the correlation of the PIK3CA mutation and $\mathrm{pCR}$ in TNBC and demonstrated that TNBC with the PIK3CA H1047R mutation is less likely to achieve pCR after AT-based NAC than TNBC without the mutation (22). However, although TP53 mutations are frequent in breast cancer, mutations do not predict the response to AT-based NAC in the TNBC and human epidermal 
Table 2 Summary of results for the risk ratio (RR) of adverse effects in the cyclophosphamide arm compared to control arm

\begin{tabular}{|c|c|c|c|c|c|c|}
\hline Outcomes & References & Participants & $\begin{array}{l}\mathrm{RR}(95 \% \mathrm{Cl}) \text {, cyclophosphamide arm } \\
\text { compared to control arm }\end{array}$ & $P$ value & \multicolumn{2}{|c|}{ Heterogeneity } \\
\hline Anemia & (3) & 1,397 & $1.14(0.35,3.69)$ & 0.82 & 0.05 & 68 \\
\hline Leucopenia & (2) & 1,848 & $3.43(0.41,28.43)$ & 0.25 & $<0.001$ & 100 \\
\hline Neutropenia & (3) & 1,932 & $1.18(0.46,3.02)$ & 0.73 & $<0.001$ & 99 \\
\hline Neutropenic fever & (2) & 1,029 & $0.41(0.00,62.18)$ & 0.73 & $<0.001$ & 96 \\
\hline Alopecia & (3) & 1,932 & $1.01(0.98,1.04)$ & 0.66 & 0.48 & 0 \\
\hline Infection & (2) & 1,848 & $0.57(0.41,0.79)$ & $<0.001$ & 0.90 & 0 \\
\hline Fatigue & (2) & 1,848 & $1.06(0.57,1.97)$ & 0.86 & 0.004 & 88 \\
\hline Skin changes & (2) & 1,848 & $0.69(0.06,8.32)$ & 0.77 & $<0.001$ & 97 \\
\hline Loss of appetite & (2) & 1,848 & $0.83(0.62,1.11)$ & 0.21 & 0.34 & 0 \\
\hline Nausea or vomiting & (3) & 1,932 & $1.51(1.11,2.06)$ & 0.009 & 0.16 & 46 \\
\hline Diarrhea & (3) & 1,932 & $0.46(0.30,0.68)$ & $<0.001$ & 0.75 & 0 \\
\hline Stomatitis & (2) & 1,848 & $1.08(0.29,4.06)$ & 0.91 & 0.002 & 90 \\
\hline Abnormal level of bilirubin & (2) & 1,029 & $0.30(0.03,2.82)$ & 0.29 & 0.92 & 0 \\
\hline Abnormal level of AST & (2) & 1,029 & $1.86(0.47,7.35)$ & 0.38 & 0.87 & 0 \\
\hline Abnormal level of ALT & (2) & 1,029 & $1.47(0.34,6.30)$ & 0.60 & 0.01 & 84 \\
\hline
\end{tabular}

Italic $\mathrm{P}$ values indicate statistical significance. $\mathrm{RR}$, risk ratio; $\mathrm{Cl}$, confidence interval; $\mathrm{AST}$, aspartate aminotransferase; $A L T$, alanine aminotransferase.

growth factor receptor-2 positive (HER2 ${ }^{+}$) subtypes (23). Therefore, genetic biomarkers provide important predictions for the efficacy of NAC in breast cancer and should be verified by further studies.

The main finding of our study was the similar rates of pCR, breast-conserving surgery and clinical response between the AT and TAC regimens. Although a lower rate of no clinical response was associated with the TAC regimen, there was equivalent efficiency between the AT and TAC regimens. According to these results, one may assume that cyclophosphamide may be unnecessary in AT-based NAC in breast cancer. However, it should be clarified that several included studies involved dose-dense regimens, prophylactic granulocyte colony-stimulating factor (G-CSF) or the addition of carboplatin in the control group, which may improve clinical outcomes compared with those of standard regimens. A dose-dense regimen is recommended by the National Comprehensive Cancer Network (NCCN) guidelines as standard chemotherapy (24). Several randomized trials have shown that dose-dense or dose-intensified chemotherapy results in superior overall survivor (OS) compared to conventionally dosed chemotherapy $(25,26)$. In addition, several randomized controlled trials have compared other anthracycline-based or taxane-based regimens for the treatment of breast cancer. Diéras et al. (27) evaluated the pCR rate of doxorubicin combined with paclitaxel (AP) and doxorubicin combined with cyclophosphamide (AC) as NAC in breast cancer patients. The pCR rate was $16 \%$ and $10 \%$ in the AP and AC arms, respectively, and breast-conserving surgery was performed in $58 \%$ and $45 \%$ of patients in the AP and $\mathrm{AC}$ arms, respectively.

Another important finding was that the TAC regimen was associated with a lower rate of infection and diarrhea but a higher rate of thrombocytopenia, sensory/motor neuropathy and nausea/vomiting than the AT regimen. The lower incidence of infection and diarrhea in the TAC 
arm may result from lower doses of chemotherapy drugs. The application of prophylactic G-CSF can enhance platelet aggregation and activation, which may account for the lower rate of thrombocytopenia in the TA arm (28). It has been reported that among the taxane-based regimens, overall, TAC is associated with the highest incidence of toxicity (29). Nabholtz et al. (30) assessed the toxicity of the TAC regimen and concluded that the most common adverse event was grade 4 neutropenia followed by febrile neutropenia and infection. Kim et al. (31) evaluated the safety of the combination of doxorubicin and cyclophosphamide (AC) in patients with breast cancer and indicated that the most frequent adverse effects included nausea, alopecia, generalized muscle weakness, myalgia, mucositis, anorexia, dyspepsia and diarrhea. van Rossum et al. (32) compared toxicity reactions between doxorubicin and cyclophosphamide (AC) and TAC regimens and showed a higher rate of anemia (18.9\% versus $4.7 \%)$ as well as a lower rate of diarrhea $(6.4 \%$ versus $16.6 \%)$ and peripheral neuropathy ( $4.6 \%$ versus $14.4 \%$ ) in the AC arm. A meta-analysis performed by Do et al. (33) revealed that febrile neutropenia occurred in 5-69\% of patients who received the combination regimen of docetaxel and cyclophosphamide and that febrile neutropenia was effectively prevented by prophylactic G-CSF. Cardiac effects such as cardiomyopathy can also be noted in AT-based NAC, though their incidence is relatively low (34).

The present study compares the efficacy and safety of TAC and AT based regimens as NAC in breast cancer, indicating cyclophosphamide may be unnecessary in AT-based regimens. These data may provide important information for clinical practice. However, the limitations of our study should be also noticed. First, only four studies are included, with a relatively small patient population. And some included studies were performed ten years ago. Second, the regimens are different among these studies, which weakens the comparison. Third, the control group involves dose-dense regimens, prophylactic G-CSF or addition of carboplatin, which may influence the clinical outcomes. Fourth, the study fails to provide information about the long-term survival rates of participants as well as the effectiveness on different subtypes. Fifth, sequential effects of anthracycline and taxane are not discussed in the present study due to lack of data. Sixth, although the difference of toxicity reactions is detected between the two groups in the study, the underlying mechanisms are not investigated.

\section{Conclusions}

AT-based NAC with or without cyclophosphamide showed similar clinical outcomes in patients with operable breast cancer. The addition of cyclophosphamide might increase the risks of thrombocytopenia, sensory/motor neuropathy and nausea/vomiting.

\section{Acknowledgments}

Funding: This work was supported by the National Natural Science Foundation of China $(81472753,81672634)$, Capital Health Development Research Project (20182-4023), National Key Research and Development Project (2018YFC0115204), and Foundation for Clinical Translational and Medical Research, Chinese Academy of Medical Sciences (12019XK320071).

\section{Footnote}

Reporting Checklist: The authors have completed the PRISMA reporting checklist. Available at http://dx.doi. org/10.21037/gs-20-593

Conflicts of Interest: All authors have completed the ICMJE uniform disclosure form (available at http://dx.doi. org/10.21037/gs-20-593). The authors have no conflicts of interest to declare.

Ethical Statement: The authors are accountable for all aspects of the work in ensuring that questions related to the accuracy or integrity of any part of the work are appropriately investigated and resolved.

Open Access Statement: This is an Open Access article distributed in accordance with the Creative Commons Attribution-NonCommercial-NoDerivs 4.0 International License (CC BY-NC-ND 4.0), which permits the non-commercial replication and distribution of the article with the strict proviso that no changes or edits are made and the original work is properly cited (including links to both the formal publication through the relevant DOI and the license). See: https://creativecommons.org/licenses/by-nc-nd/4.0/.

\section{References}

1. Torre LA, Bray F, Siegel RL, et al. Global cancer statistics, 2012. CA Cancer J Clin 2015;65:87-108. 
2. Bray F, Ferlay J, Soerjomataram I, et al. Global cancer statistics 2018: GLOBOCAN estimates of incidence and mortality worldwide for 36 cancers in 185 countries. CA Cancer J Clin 2018;68:394-424.

3. Torre LA, Islami F, Siegel RL, et al. Global Cancer in Women: Burden and Trends. Cancer Epidemiol Biomarkers Prev 2017;26:444-57.

4. Frei E. Clinical cancer research: an embattled species. Cancer 1982;50:1979-92.

5. Read RL, Flitcroft K, Snook KL, et al. Utility of neoadjuvant chemotherapy in the treatment of operable breast cancer. ANZ Journal of Surgery 2015;85:315-20.

6. Rastogi P, Anderson SJ, Bear HD, et al. Preoperative chemotherapy: updates of National Surgical Adjuvant Breast and Bowel Project Protocols B-18 and B-27. J Clin Oncol 2008;26:778-85.

7. Bear HD, Anderson S, Brown A, et al. The effect on tumor response of adding sequential preoperative docetaxel to preoperative doxorubicin and cyclophosphamide: preliminary results from National Surgical Adjuvant Breast and Bowel Project Protocol B27. J Clin Oncol 2003;21:4165e4174.

8. De Laurentiis M, Cancello G, D'Agostino D, et al. Taxane-based combinations as adjuvant chemotherapy of early breast cancer: a meta-analysis of randomized trials. J Clin Oncol 2008;26:44-53.

9. Singh JC, Mamtani A, Barrio A, et al. Pathologic Complete Response with Neoadjuvant Doxorubicin and Cyclophosphamide Followed by Paclitaxel with Trastuzumab and Pertuzumab in Patients with HER2-Positive Early Stage Breast Cancer: A Single Center Experience. Oncologist 2017;22:139-43

10. Caparica R, Bruzzone M, Poggio F, et al. Anthracycline and taxane-based chemotherapy versus docetaxel and cyclophosphamide in the adjuvant treatment of HER2-negative breast cancer patients: a systematic review and meta-analysis of randomized controlled trials. Breast Cancer Res Treat 2019;174:27-37.

11. Schulz KF, Altman DG, Moher D, et al. CONSORT 2010 Statement: updated guidelines for reporting parallel group randomised trials. PLoS Med 2010;7:e1000251.

12. Jackisch C, von Minckwitz G, Eidtmann H, et al. Dose-dense biweekly doxorubicin/docetaxel versus sequential neoadjuvant chemotherapy with doxorubicin/ cyclophosphamide/docetaxel in operable breast cancer: second interim analysis. Clin Breast Cancer 2002;3:276-80.

13. von Minckwitz G, Raab G, Caputo A, et al. Doxorubicin with cyclophosphamide followed by docetaxel every 21 days compared with doxorubicin and docetaxel every 14 days as preoperative treatment in operable breast cancer: the GEPARDUO study of the German Breast Group. J Clin Oncol 2005;23:2676-85.

14. Hong WS, Jeon JY, Kang SY, et al. Comparison of neoadjuvant adriamycin and docetaxel versus adriamycin, cyclophosphamide followed by paclitaxel in patients with operable breast cancer. J Korean Surg Soc 2013;85:7-14.

15. Schneeweiss A, Möbus V, Tesch H, et al. Intense dose-dense epirubicin, paclitaxel, cyclophosphamide versus weekly paclitaxel, liposomal doxorubicin (plus carboplatin in triple-negative breast cancer) for neoadjuvant treatment of high-risk early breast cancer (GeparOcto-GBG 84): A randomised phase III trial. Eur J Cancer 2019;106:181-92.

16. Crown J, O'Leary M, Ooi WS. Docetaxel and paclitaxel in the treatment of breast cancer: a review of clinical. Oncologist 2004;9:24-32.

17. Andrade JM, Carrara HH, Pimentel FF, et al. Taxane-based chemotherapy enhances response to neoadjuvant treatment for stage II and III breast cancer. Med Oncol 2011;28:S65-9.

18. Yoon HK, Kim TH, Park S, et al. Effect of anthracycline and taxane on the expression of programmed cell death ligand-1 and galectin-9 in triple-negative breast cancer. Pathol Res Pract 2018;214:1626-31.

19. Javeed A, Ashraf M, Riaz A, et al. Paclitaxel and immune system. Eur J Pharm Sci 2009;38:283-90.

20. Melichar B, Touskova M, Dvorak J, et al. The peripheral blood leukocyte phenotype in patients with breast cancer: effect of doxorubicin/paclitaxel combination chemotherapy. Immunopharmacol Immunotoxicol 2001;23:163-73.

21. Bignon L, Fricker JP, Nogues C, et al. Efficacy of anthracycline/taxane-based neo-adjuvant chemotherapy on triple-negative breast cancer in BRCA1/BRCA2 mutation carriers. Breast J 2018;24:269-77.

22. Guo S, Loibl S, Minckwitz GV, et al. PIK3CA H1047R Mutation Associated with a Lower Pathological Complete Response Rate in Triple-Negative Breast Cancer Patients Treated with Anthracycline-Taxane-Based Neoadjuvant Chemotherapy. Cancer Res Treat 2020;52:689-96.

23. Darb-Esfahani S, Denkert C, Stenzinger A, et al. Role of TP53 mutations in triple negative and HER2-positive breast cancer treated with neoadjuvant anthracycline/ taxane-based chemotherapy. Oncotarget 2016;7:67686-98.

24. National Comprehensive Cancer Network Guidelines. Available online: https://www.nccn.org/professionals/ 
physician_gls/pdf/breast.pdf

25. Sparano JA, Wang M, Martino S, et al. Weekly paclitaxel in the adjuvant treatment of breast cancer. N Engl J Med 2008;358:1663e71.

26. Del Mastro L, De Placido S, Bruzzi P, et al. Flourouracil and dose-dense chemotherapy in adjuvant treatment of patients with early-stage breast cancer: an open-label, 2 × 2 factorial, randomized phase 3 trial. Lancet 2015;385:1863-72.

27. Diéras V, Fumoleau P, Romieu G, et al. Randomized parallel study of doxorubicin plus paclitaxel and doxorubicin plus cyclophosphamide as neoadjuvant treatment of patients with breast cancer. J Clin Oncol 2004;22:4958-65.

28. Spiel AO, Bartko J, Schwameis M, et al. Increased platelet aggregation and in vivo platelet activation after granulocyte colony-stimulating factor administration. A randomised controlled trial. Thromb Haemost 2011;105:655-62.

29. Loibl S, von Minckwitz G, Harbeck N, et al. Clinical feasibility of (neo)adjuvant taxane-based chemotherapy in older patients: analysis of $>4,500$ patients from four German randomized breast cancer trials. Breast Cancer Res 2008;10:R77.

Cite this article as: Kang YK, Si YR, An GY, Yuan P. Efficacy and safety of cyclophosphamide in anthracycline- and taxane-based neoadjuvant chemotherapy in breast cancer: a meta-analysis. Gland Surg 2021;10(1):252-261. doi: 10.21037/gs20-593
30. Nabholtz JM, Smylie M, Mackey JR, et al.

Docetaxel/doxorubicin/cyclophosphamide in the treatment of metastatic breast cancer. Oncology (Williston Park) 1997;11:37-41.

31. Kim GM, Kim JH, Kim JH, et al. A Phase II Study to Evaluate the Safety and Efficacy of Pegteograstim in Korean Breast Cancer Patients Receiving Dose-Dense Doxorubicin/Cyclophosphamide. Cancer Res Treat 2019;51:812-8.

32. van Rossum AGJ, Kok M, van Werkhoven E, et al. Adjuvant dose-dense doxorubicin-cyclophosphamide versus docetaxel-doxorubicin-cyclophosphamide for high-risk breast cancer: First results of the randomised MATADOR trial (BOOG 2004-04). Eur J Cancer 2018;102:40-8.

33. Do T, Medhekar R, Bhat R, et al. The risk of febrile neutropenia and need for G-CSF primary prophylaxis with the docetaxel and cyclophosphamide regimen in earlystage breast cancer patients: a meta-analysis. Breast Cancer Res Treat 2015;153:591-7.

34. Valero V, Perez E, Dieras V. Doxorubicin and taxane combination regimens for metastatic breast cancer: focus on cardiac effects. Semin Oncol 2001;28:15-23. 


\section{Supplementary}

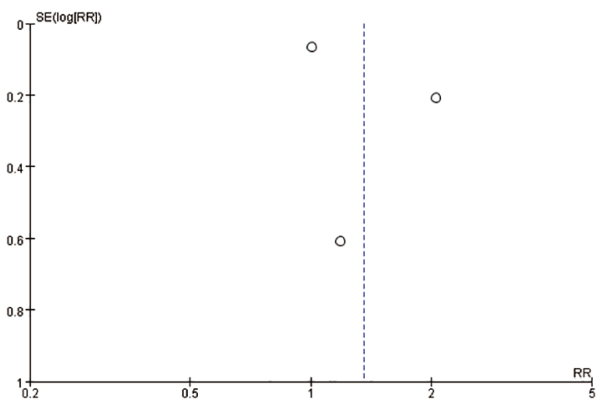

Figure S1 Funnel plot showing the test for publication bias of pooled risk ratio (RR) with standard error (SE) of pCR rate. pCR, pathologic complete response. 